\title{
Valoración de la capacidad laboral de la malformación de Arnold Chiari tipo I
}

\section{Valuation of work disability of the Arnold Chiari malformation}

\section{Raúl Jesús Regal Ramos}

Unidad Médica del Equipo de Valoración de Incapacidades. Instituto Nacional de la Seguridad Social Madrid. España.

Recibido: 03-12-10

Aceptado: 10-02-11

\section{Correspondencia}

Raúl Jesús Regal Ramos

Unidad Médica del Equipo de Valoración de Incapacidades

Lope de Hoyos, 169

28002 Madrid. España

Tfno: 915907432

E-mail: raul-jesus.regal@inss.seg.social

Resumen

Introducción: La reciente publicación de un documento de consenso por el Instituto de Investigación de Enfermedades Raras, el aumento de la incidencia de malformación de Arnold - Chiari (por la cada vez más frecuente solicitud de RMN cervicales) y, paralelamente, el aumento de la presencia de estos pacientes en las consultas de los médicos encargados de valorar su capacidad funcional, nos invita a hacer una reflexión sobre la valoración laboral de estos pacientes

Objetivos: Esta revisión pretende realizar un acercamiento a distintos aspectos relevantes en la valoración de la discapacidad laboral de estos pacientes

Metodología: Se han revisado hasta Mayo de 2010 las siguientes bases de datos bibliográficas: Medline, Embase, Cochrane.

Resultados: La intensidad de los síntomas (sobre todo dolor) no está en relación directa con la gravedad de las lesiones observadas en las pruebas de imagen.

El cuadro clínico puede ser fluctuante, con períodos de agudización y de remisión.

Los síntomas que mejor responden a la cirugía son los síntomas debido a compresión del tronco cerebral (especialmente la cervicocefalalgia), desapareciendo en algunos casos.

Son factores de mala evolución postoperatoria la presencia en el examen prequirúrgico de atrofia, ataxia, escoliosis y que el tiempo entre el inicio de los síntomas y la cirugía haya sido superior a 2 años.

Aquellas manifestaciones clínicas que no desaparecen en el postoperatorio ni en el periodo de seguimiento podrían estar relacionadas con el daño permanente de las vías nerviosas o de sus núcleos.

Pese a tratarse de una enfermedad congénita podría tener la consideración de accidente de trabajo, si los síntomas se desencadenan tras un traumatismo.

La evidencia de la eficacia del tratamiento para el dolor resulta escasa.

Conclusiones: La evaluación de estos pacientes debe ser siempre individualizada, considerando las limitaciones orgánicas y/o funcionales, y relacionándolas con los requerimientos del puesto de trabajo, no obstante existen una serie de aspectos comunes relevantes en la valoración laboral de estos pacientes.

Med Segur Trab (Internet) 2011; 57 (222) 95-100

Palabras clave: Malformación de Chiari. Discapacidad laboral. Repercusión Funcional. 


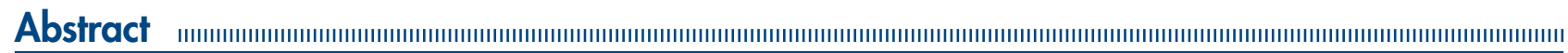

Introduction: The recent publication of a consensus document by the Research Institute of Rare Diseases, the increased incidence of Arnold-Chiari malformation (by the increased demand of cervical MRI) and, simultaneously, the increased presence of these patients in hospitals where doctors can assess their functional capacity, invites us to reflect on the assessment of the working disability of these patients.

Objectives: This review pretends to make an approach to various relevant aspects on the assessment of these patients' working disability

Material and methods: The following bibliographical database have been reviewed until May 2010: Medline, Embase, Cochrane.

Results: The intensity of the symptoms (mostly pain) is not directly related to the severity of the lesions observed in the imaging tests.

The clinical symptoms may fluctuate, with periods of exacerbation and remission.

The symptoms that best respond to surgery are those due to compression of the brainstem (especially cervical headache), disappearing in some cases.

The presence of atrophy, ataxia, scoliosis in the pre surgery tests, and more than two years between the beginning of the symptoms and the surgery are poor post surgery evolution factors.

Those clinical manifestations that do not disappear in the post surgery period or in the follow-up period could be related to permanent damage of the neural pathways or their nuclei. trauma.

Despite being a congenital disease, it could be treated as a working accident, if symptoms appear after

Evidence of the effectiveness of pain treatment is low.

Conclusions: These patients' evaluation should always be individualized, considering the organic and or functional limitations and relating them to the requests of their job. Nevertheless, there exists a series of common relevant aspects in these patients' working assessment.

Med Segur Trab (Internet) 2011; 57 (222) 95-100

Key words: Chiari I malformation. Work disability. Functional impact. 


\section{INTRODUCCIÓN}

Bajo el término de Malformación de Chiari se agrupan una serie de anomalías congénitas de la base del cráneo caracterizadas fundamentalmente por descenso de las amígdalas cerebelosas por detrás del bulbo y médula espinal en el canal cervical a través del agujero occipital (Figura1) y, desplazamiento del bulbo hacia el canal cervical con la parte inferior del cuarto ventrículo.

Figura 1. Descenso de $5 \mathrm{~mm}$. de las amígdalas cerebelosas por debajo del foramen magnum.

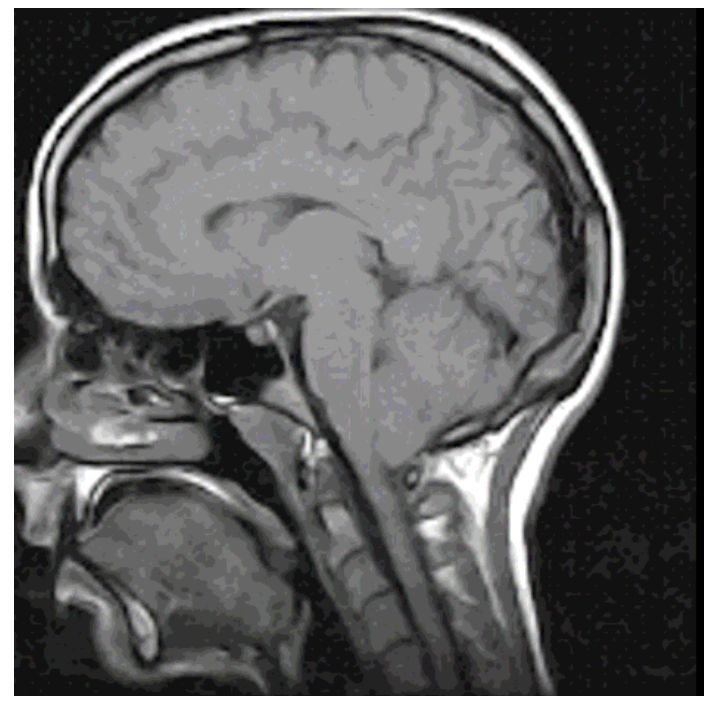

Aunque existen diversas teorías, no hay ninguna universalmente aceptada que explique el desarrollo de una malformación de Chiari.

Existen 5 subtipos ( 0 a IV) de malformación de Chiari. En el tipo 0 existen solo una alteración de la hidrodinámica del LCR a nivel del foramen mágnum con mínimos datos de herniación amigdalar o sin ellos. El tipo I es el más frecuente y es al que haremos referencia en este trabajo, es la denominada malformación de Chiari del adulto. En el tipo I se produce herniación caudal de las amígdalas cerebelosas mayor de $5 \mathrm{~mm} .{ }^{1}$ por debajo del foramen mágnum y aunque se asocia a siringomielia en el 50\% de los casos, no suelen existir otras anomalías cerebrales asociadas (no suele acompañarse de descenso del tronco del encéfalo o del $4^{\circ}$ ventrículo y la hidrocefalia solo aparece en el 3-10\% de los casos). El tipo II suele debutar en la infancia y se asocia siempre con espina bífida abierta e hidrocefalia. El tipo III generalmente es incompatible con la vida y por último el tipo IV asocia aplasia o hipoplasia del cerebelo

La técnica diagnóstica de elección es la resonancia magnética ${ }^{2}$. La RM, pone de manifiesto no sólo la anomalía de la unión cráneo-vertebral, sino también su intensidad, la repercusiones sobre las estructuras vecinas y la presencia de siringomielia

El único tratamiento eficaz es el quirúrgico y esta indicado cuando la progresión o intensidad de los síntomas provocan una incapacidad significativa ${ }^{3}$ El tratamiento de elección para estos pacientes es la craniectomía descompresiva suboccipital (que suele ampliarse con una laminectomía de C1 y C2) con apertura de la duramadre y colocación de una plastia de dura para ampliar el espacio de la fosa posterior. Cuando asocia hidrocefalia debe implantarse una derivación de LCR

Los pacientes asintomáticos sin siringomielia no son candidatos a cirugía, mientras que en los que presentan siringomielia se recomienda la cirugía cuando la clínica progresa o la RM demuestra aumento del crecimiento del quiste

El tratamiento no quirúrgico abarca el tratamiento del dolor y la terapia de rehabilitación. Si la evidencia del tratamiento farmacológico para el dolor resulta escasa (anticonvulsivantes, anestésicos locales, antidepresivos, opiáceos y otros analgésicos), la 
del tratamiento no farmacológico, como la estimulación la estimulación eléctrica medular o periférica, resulta inexistente. El tratamiento rehabilitador va encaminado a aliviar el dolor, mejorar la amplitud del recorrido articular, normalizar el tono, reducir la espasticidad, reeducar las reacciones de equilibrio y de la marcha, disminuir los problemas orofaciales, como disfonía, disartria y disfagia,..

\section{VALORACIÓN LABORAL}

La valoración de estos pacientes debe ser siempre individualizada, evaluando cada síntoma y la intensidad de estos, pero vamos a realizar un acercamiento a los distintos aspectos relevantes en la valoración laboral de estos pacientes.

\section{Valoración de la clínica}

La intensidad de los síntomas (sobre todo el dolor) no está en relación directa con la gravedad de las lesiones observadas en las pruebas de imagen ${ }^{4}$.

Existe mucha variabilidad clínica entre los pacientes pudiendo presentarse como un hallazgo casual, con manifestaciones clínicas inespecíficas o con signos neurológicos graves.

El inicio suele ser insidioso y progresivo. La cefalea suboccipital es el síntoma y la forma de presentación más frecuente (60-70\% de los casos). Las alteraciones sensitivas en miembros superiores, sobre todo parestesias, son el segundo síntoma en frecuencia.

Además debemos tener en cuenta que el cuadro clínico puede ser fluctuante, con períodos de agudización y de remisión.

En aquellos pacientes asintomáticos a los que se les realiza una RMN cervical por otro motivo (afectación radicular por hernia discal, para descartar fractura,..) y se les descubre como hallazgo casual la presencia de esta malformación en un grado leve, probablemente no deberemos plantearnos la incapacidad laboral.

Valoraremos también el impacto psíquico de esta patología y su grado, ya que con frecuencia es necesario el apoyo de un especialista.

El conocimiento fisiopatológico de la clínica es necesario para realizar una correcta valoración laboral:

- Los síntomas secundarios a alteraciones de la dinámica del LCR (cefalea suboccipital, cervicalgia, mareos, vértigos, acúfenos, hipoacusia, diplopia, fotopsias, visión borrosa, fotofobia,..) aparecen o empeoran al realizar esfuerzos (defecación, tos, estornudo, ejercicio físico, etc.). Las cervicalgias, se caracterizan por carecer de distribución radicular y estar acompañadas de molestias continuas, urentes y profundas, localizadas en hombros, nuca o miembros superiores y que típicamente aumentan con maniobras de Valsalva

- Entre los síntomas secundarios a compresión afectación cerebelosa tenemos la ataxia, nistagmus vertical hacia abajo, temblor, alteración de la coordinación ${ }^{5}$. La posturografía dinámica permite evaluar objetivamente los trastornos del equilibrio.

- Los síntomas secundarios a compresión de médula y de bulbo incluyen afectación de primera neurona motora y segunda neurona motora, afectación de la sensibilidad, e incluso en casos muy avanzados puede haber afectación de esfínteres.

- Síntomas secundarios a afectación de pares craneales bajos (se afectan en 15-25\% de casos) como disartria, disfonía, parálisis de cuerdas vocales, disfagia, ausencia de reflejo nauseoso, neuralgia del trigémino o del glosofaríngeo, bradicardia sinusal. 
- Los síntomas secundarios a siringomielia (aproximadamente el 90\% de los casos de siringomielia ocurren en la malformación de Chiari tipo I, mientras que sólo un $50 \%$ de los pacientes con malformación de Chiari tipo I tienen asociada siringomielia) incluyen síntomas de síndrome centromedular (disociación termoalgésica suspendida con amiotrofia braquial) y/o de siringomielia a nivel del bulbo o siringobulbia (parálisis del paladar o de las cuerdas vocales, disartria, nistagmo horizontal o vertical, mareos episódicos, debilidad o amiotrofia lingual, la hipoalgesia trigeminal)

\section{Valoración del tratamiento}

Valoraremos las limitaciones derivadas de la cirugía (por la presencia de válvulas ventrículo-peritoneales, de artrodesis occipito-cervicales,..) y las derivadas del tratamiento farmacológico (opiáceos, anticonvulsivantes, psicofármacos,..).

Debemos tener en cuenta que el único tratamiento eficaz es el quirúrgico y esta indicado cuando la progresión o intensidad de los síntomas provocan una incapacidad significativa. Los pacientes asintomáticos sin siringomielia no son candidatos a cirugía, mientras que en los que presentan siringomielia se recomienda la cirugía cuando progresan la clínica o las alteraciones en la RMN.

La eficacia de los analgésicos es escasa.

El tratamiento rehabilitados va encaminado además de a aliviar el dolor, a mejorar el recorrido articular, reeducar equilibrio y marcha, disminuir disfonía, disartria, disfagia,..

\section{Valoración del pronóstico}

Los síntomas que mejor responden a la cirugía son los síntomas debidos a compresión del tronco cerebral (especialmente la cervicocefalalgia), desapareciendo en algunos $\operatorname{casos}^{6} \mathrm{y}$, también, el síndrome cerebeloso. Por tanto, cuando estos síntomas sean la principal causa de la limitación laboral de estos pacientes, debemos valorarlos tras la cirugía.

También debemos tener presente que aquellas manifestaciones clínicas que no desaparecen en el postoperatorio ni en el periodo de seguimiento podrían estar relacionadas con el daño permanente de las vías nerviosas o de sus núcleos ${ }^{7}$.

Son factores de mala evolución postoperatoria: la presencia en el examen prequirúrgico de atrofia, ataxia, escoliosis y el tiempo entre el inicio de los síntomas y la cirugía (si es superior a 2 años puede hacer que los signos derivados de la compresión de estructuras de la fosa posterior sean relativamente resistentes al tratamiento). Pese a que como hemos visto, la clínica mejora tras la cirugía, algunos autores como Rhoton ${ }^{8}$, sostienen que el mayor beneficio de la cirugía es detener la progresión de la patología

\section{Escalas}

En casos muy graves debemos aplicar diversas escalas que nos orienten sobre la necesidad de terceras personas para la realización de ABVD: Barthel, Katz, Lawton, FIM, Nottingham, etc,.. El balance muscular podemos graduarlo con la escala de Daniels.

\section{Otros aspectos a valorar}

Aunque el Chiari tipo I puede ser asintomático de por vida, si después de un traumatismo se desencadenan los síntomas, estos han sido considerados por algunos jueces como originados por el traumatismo, lo cual implica que pese a tratarse de una enfermedad congénita podría tener la consideración de accidente de trabajo. 
Profesiones sujetas a determinados requerimientos psicofísicos establecidos por normativa (pilotos, conductores profesionales,..), deberán permanecer en IT hasta que cumplan éstos requisitos.

Para concluir, debemos tener presente que la valoración de la capacidad laboral de un trabajador requiere objetivar las limitaciones orgánicas y/o funcionales, pero también resulta imprescindible conocer los requerimientos del puesto de trabajo, con objeto de poder determinar si las limitaciones impiden al trabajador desarrollar su actividad labora. Esta revisión recoge los aspectos más relevantes de la valoración de la discapacidad laboral de los pacientes con Chiari tipo I.

\section{REFERENCIAS BIBLIOGRÁFICAS}

1. Payner TD, Prenger E, Berger TS, Crone KR. Acquired Chiari Malformations: incidence, diagnosis, and management. Neurosurgery. 1994;34:429-34

2. Ventureyra EC, Asis HA, VassilyadiM: The role of cine flor MRI in children with Chiari I malformation. Childs Nerv Syst 2003. 19:109-13

3. Martínez-Vila E, Riverol Fernández M, Irimia Sieira P. Síndrome vestibular central. REV MED UNIV NAVARRA/VOL 47, $\mathrm{N}^{\circ} 4,2003,51-59$

4. http://www.semergen.es/semergen/microsites/manuales/consenso_chiari

5. Bell W. et al: Symptomatic Arnold Chiari malformation: review of experience with 22 cases. J of Neurosurg 66, 812-816, 1987

6. Bindal AK, Dunsker SB, Tew JM. Chiari I malformation: classification and management. Neurosurgery 1995; 37(6): 1069-74

7. Alvarez-Betancourt,L; García-Rentaria, JA; Lopez- Ortega,SJ; Caldera-Duarte,A.: Malformación de Chiari tipo I: evolución postoperatoria a 2 años. Análisis de 10 casos. Neurocirugía 2005; 16:34-38

8. Rothon A. et al: Mycrosurgery of siringomielia and syringolielic cor syndrom. En Schmidek,H.H and Sweet,W.H.,ed W.B. Saunders Co. Philadelphia 1995.2: 1745 - 1764

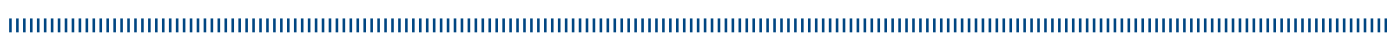

